\title{
ULTRAVIOLET ABSORPTION BY INTERSTELLAR GAS AT LARGE DISTANCES FROM THE GALACTIC PLANE
}

Blair D. Savage and Klaas S. de Boer University of Wisconsin, Madison, WI 53706, U.S.A.

During the past two years we have acquired a total of 18 high dispersion spectra of 6 stars in the LMC, 3 in the SMC, and 2 foreground galactic stars. The LMC stars observed are HD 36402, HD 268605, Sk, 18-67, HD 38268, HD 38282, and HD 269357. The SMC stars observed are Sk 80 , Sk 108, and HD 5980.

Various investigations undertaken with these spectra have resulted in the following publications:

1. "Observational Evidence for a Hot Gaseous Galactic Corona" Savage, B.D. and de Boer, K.S. 1979, Ap.J. Letters, 230, L77.

2. "Ultraviolet Absorption by Interstellar Gas Near 30 Doradus" de Boer, K.S., Koornneef, J., and Savage, B.D. 1980, Ap.J., (March 15).

3. "Evidence for Hot Gaseous Coronae Around the Magellanic Clouds" de Boer, K.S., and Savage, B.D. 1980, Ap.J., (May 15).

4. 'Ultraviolet Absorption by Interstellar Gas At Large Distances from the Galactic Plane"

Savage, B.D., and de Boer, K.S., Ap.J., (submitted).

Paper 4 contains a reasonably complete discussion of the Milky Way absorption features toward the 9 Magellanic cloud stars. Preprints of this paper are available on request; the abstract is given below:

Abstract from "Ultraviolet Absorption by Interstellar Gas at Large Distances from the Galactic Plane"', Savage and de Boer, Ap.J., (submitted):

We have analyzed 18 high-dispersion IUE spectra of 6 stars in the LMC, 3 stars in the SMC, and 2 foreground stars. Fourteen spectra cover the wavelengths $\lambda \lambda 1150-2000 \AA$ and 4 cover $\lambda \lambda 1900-3200 \AA$. The velocity resolution is $\sim 25 \mathrm{~km} \mathrm{~s}^{-1}$. All the Magellanic Cloud star spectra exhibit exceedingly strong interstellar absorption lines due to a wide range of ionization stages at galactic velocities and at velocities associated with the LMC or SMC. In this paper the analysis is restricted to the Milky Way absorption features. Toward the LMC stars, the strong interstellar lines have a positive velocity extension to $V_{L S R} \sim 150 \mathrm{~km} \mathrm{~s}-1$, which exceeds by $\sim 100 \mathrm{~km} \mathrm{~s}^{-1}$ the extension recorded toward the SMC stars. The most straightforward interpretation of these velocity extensiuns is obtained by assuming that gas at large distances away from the plane of the galaxy participates in the rotation of the galaxy as found in the galactic disk. This then indicates that we have detected absorption by gas as far as perhaps 10 to $15 \mathrm{kpc}$ below the plane of the galaxy. Toward many of the LMC stars the low ion stage lines exhibit a component structure with strong features near 60 and $130 \mathrm{~km} \mathrm{~s}^{-1}$. The presence 
of 0 I absorption at these velocities implies that a portion of the gas is neutral even though it is not detected in existing $21-\mathrm{cm}$ emission data. A curve of growth analysis provides some information on the composition of the low-ion high-velocity gas. Solar abundances are consistent with the uncertain abundance estimates, although there is the possibility that the $\mathrm{N} / 0$ ratio is less than solar. The high-ionization lines of $\mathrm{Si}$ IV and $\mathrm{C}$ IV are strong toward all the Magellanic Cloud stars while $\mathrm{N} \mathrm{V}$ is not detected. These data provide definitive proof that highly ionized species exist in the general interstellar medium of our galaxy away from local stellar environments. The weakness of the high-ion lines toward the foreground galactic stars implies that the strong absorption toward the extragalactic stars is primarily produced by gas beyond $r \sim 0.9 \mathrm{kpc}$. For this halo gas we obtain toward the LMC $\log N(C$ IV) $\sim 14.0, \log N(S i$ IV $) \sim 13.5$, and $\log N(N V)<13.5$. Toward the SMC the N V limit is the same but the C IV and Si IV column densities are at least a factor of 2 larger. If the high-ion gas is produced by equilibrium "coronal ionization" the implied temperatures are in the range 0.7 to $1.4 \times 105 \mathrm{~K}$. The high-ion line profiles contain less component structure than the low ion profiles, suggesting a smoother high-ion gas distribution. While the low-ion gas might represent condensed material in a galactic fountain model, it is not obvious where within this model the high-ion gas fits since the fountain, if it exists, is presumably driven by gas with much higher temperatures. If the high-ion gas is primarily formed in "cloud coronae" one might expect to find a better correspondence between the low- and high-ion line profiles than is observed. Perhaps the high-ion gas represents; a type of "transition region" between gas in the plane of the galaxy and a hotter exterior zone that cannot be studied with IUE data. Assuming solar abundances, the amount of halo gas detected represents $\sim 2$ percent of that found in the galactic disk. 